\title{
Heat transfer and solidification processes of alloy melt with undercooling_Part I: Experimental results
}

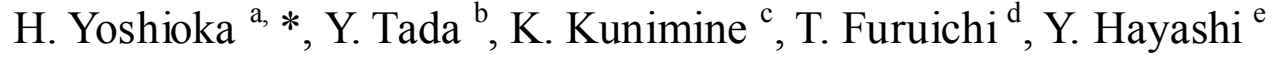 \\ a Department of Maritime Technology, Toyama National College of Maritime Technology, \\ 1-2 Ebie Neriya, Shinminato, Toyama 933-0293, JAPAN \\ ${ }^{\mathrm{b}}$ Department of Human and Mechanical Systems Engineering, Kanazawa University, \\ 2-40-20 Kodatsuno, Kanazawa, Ishikawa 920-8667, JAPAN \\ ${ }^{\mathrm{c}}$ Department of Mechanical Engineering, Akashi National College of Technology, 679-3 \\ Nishioka, Uozumi-cho, Akashi, Hyogo 674-8501, JAPAN \\ ${ }^{\mathrm{d}}$ Hitachi Cable, Ltd., 3-1-1 Sukegawa-cho, Hitachi, Ibaraki 317-0065, JAPAN

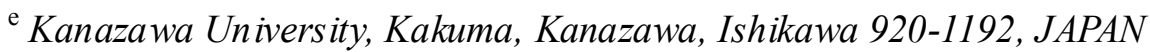

Keywords : Solidification; Undercooling; Metal \& Alloys; Crystal growth; Micro structure; Heat transfer

Corresponding author:

Hideaki Yoshioka* (As sociate Professor)

Tel.: +81-766-86-5235; Fax: +81-766-86-5110.

E-mail: yoshi@toyama-cmt.ac.jp (H. Yoshioka) 


\begin{abstract}
The solidification process of $\mathrm{Pb}-\mathrm{Sn}$ and $\mathrm{Bi}-\mathrm{Sn}$ alloy melts is discussed for a basic understanding of the essential phenomena of solidification with undercooling. First, by macroscopic observations, it is shown that the solidification process consists of the following three stages: (1) free growth with recalescence dissipation of thermal undercooling, (2) fattening of crystals with the relaxation of constitutional undercooling or with the recovering process of interrupted quasi-steady heat conduction, and (3) equilibrium solidification. The specific features of free growth under non-uniform undercooling are also shown by comparison with the Lipton, Glicksman and Kurz (LGK) model. Next, by microscopic observations, the distribution of the solute concentration and the change of crystal morphology in the solidified materials were investigated quantitatively using scanning electron microscopy and energy dispersive spectroscopy (SEM/EDS). Finally, the solidification path during the above three fundamental processes is dynamically represented on phase diagrams.
\end{abstract}




\section{INTRODUCTION}

Solidification phenomena with undercooling underlie many important scientific and engineering studies in technological applications, especially in metal and alloy process ing. The motivation for this work is to obtain a basic understanding of the mechanism and kinetics of crystal growth and the resulting microstructure in conventional solidification processing such as casting or welding. The microstructure resulting from undercooling may be important in relation to the surface properties of cast alloys. We expect that this work will lay the foundation for the transport theory of solidification with an unstable mushy zone. The solidification of alloys is always accompanied by a mushy zone. This region is generally treated as a problem of heat conduction with a phase change over a range of temperatures. The authors investigated the growth and morphology of crystals in the mushy zone, and also studied the link between heat transfer and microstructures in the mushy zone [1]. It may be assumed that the thermodynamically unstable state in front of a planar interface, which creates a rough interface, results in the formation of the mushy zone as a stable state. However, in general, it may be more reasonable to think that the mushy zone originates from the initial undercooling, which appears necessary for nucleation. When pursuing high-lebel heat transfer control in material processing, it is essential to have a more precise treatment that considers the complex solidification process in which the thermodynamically unstable undercooling field collapses by solidification and shifts to a stable system.

The solidification process of aqueous mixtures that have a diffusion distribution coefficient $k_{0}$ nearly equal to zero is schematically shown in Fig. 1. After the appearance of the non-uniform undercooling state (1), free growth initiated by heterogeneous nucleation on the cold wall rapidly occurs in the undercooled region with the rise in temperature (2-1). During this time, the initial undercooling state is almost completely replaced by the equilibrium state, since the dendritic crystals can capture the concentrated solution in the small arm spaces. Next, the width of these dendrites continuously grows from the root to the tip according to the temperature drop from external cooling (2-2). After the fattening of each crystal reaches the tip of the pre-formed 
dendrite, the stagnant solidification front (i.e., the dendrite tip) begins to advance again. These phenomena seem to occur with the interruption of the heat conduction process in the quasi-steady state. Finally, solidification proceeds in the quasi-steady state and is controlled by the heat conduction due to external cooling (3). In summary, the solidification of mixtures can be characterized by the following three stages: free growth in the undercooling state (1st stage), recovery of the interrupted heat conduction field with crystal fattening (2nd stage), and quasi-steady solidification (3rd stage). These solidification processes have been closely studied in $\mathrm{H}_{2} \mathrm{O}-\mathrm{NaCl}$ aqueous solutions [2-4].

In the solidification of alloy melts, the solute is not completely rejected. It is included to some extent in the solid when the distribution coefficient is less than unity. This induces not only microsegregation but also other behaviors that are different from that for aqueous solutions. The following are the most interesting issues in the solidification of undercooled metallic alloy melts:

(1) the fundamental solidification processes of alloy melts

(2) the characteristics of the free growth under non-uniform undercooling

(3) the microstructure and microsegregation in the free growth, and the fattening processes.

This paper discusses an experimental study of the solidification of undercooled alloy melts using $\mathrm{Pb}-\mathrm{Sn}$ and $\mathrm{Bi}-\mathrm{Sn}$ alloys. By temperature measurements and microscopic observations of the structure and composition, the redistribution of the solute concentration and the change of morphology were investigated quantitatively. By synthesizing these results with the fundamental processes of solidification, the solidification path involving the mechanism of solute redistribution is presented on phase diagrams. Microsegregation is also clarified in relation to the appearance of the solid morphology.

\section{EXPERIMENTS}


In order to discuss the solidification phenomena as a subject common to many binary alloys which are accompanied by dendrite growth in the initial process, two experiments were conducted from both the macro- and microscopic viewpoint using $\mathrm{Pb}-\mathrm{Sn}$ and $\mathrm{Bi}-\mathrm{Sn}$, respectively.

\subsection{Macroscopic observation}

First, experiments for macroscopic observation were conducted in order to clarify the solidification process of undercooled alloy melt. Sn-rich $\mathrm{Pb}-\mathrm{Sn}$ alloys were used as the samples because it was easy comparatively to observe the advancing solidification front. Figure 2(a) shows a schematic of the experimental apparatus. The mold was a cylinder of $10-\mathrm{mm}$ inside diameter and 40-mm height. The side wall was made of fused silica to allow visualization. The bottom was made of copper. The bottom surface, which was the cold wall in contact with the sample, was finished with consideration of the specularity in order to suppress heterogeneous nucleation and to obtain the large undercooling. Temperatures were measured using K-type thermocouples of $0.2-\mathrm{mm}$ diameter, which were set in the side wall at 3-mm intervals upward from the cold wall. The movement of the solidification front was observed with a video camera facing the side of the sample.

\subsection{Microscopic observation}

In order to investigate the solute concentration distribution in the solid solution, another experiment was carried out using $\mathrm{Sn}$-rich $\mathrm{Bi}-\mathrm{Sn}$ alloys, which have a larger distribution coefficient than that of $\mathrm{Pb}$-Sn. As shown in Fig. 2(b), a hollowed-out ceramic mold with inner dimensions $8 \mathrm{~mm}$ in wid th, $15 \mathrm{~mm}$ in length, and $12 \mathrm{~mm}$ in depth was used. Thermocouples were installed in the inner wall of the mold at 2-mm intervals. The setup, except for the mold, was similar to the $\mathrm{Pb}-\mathrm{Sn}$ experiment. The microstructure and microsegregation of the specimens after solidification were analyzed by a scanning electron microscope (SEM). 


\section{RESULTS AND DISCUSSION}

\subsection{Solidification process}

The solidification of an alloy melt is discussed here for a basic understanding of the essential phenomena occurring due to internal undercooling and external heat transfer cooling. Figure 3(a)-(d) shows the following for $\mathrm{Sn}-10 \mathrm{wt} \% \mathrm{~Pb}$ : (a) the sequence of the advancing solidification front, (b) microstructures observed with an optical microscope, (c) the temperature distribution at the solidification start, and (d) time changes of the advancing solidification front and temperature at the cold wall. In Fig. 3, $z$ is the distance from the cold wall, $T$ is the temperature at the solidification start, $z_{l}$ is the position of solidification front, $T_{w}$ is the temperature at the cold wall, and $t$ is the time after the cooling start.

In Fig. 3(a), the dark part is the melt and the bright part is the phase solidified. This is determined from the difference of the light reflection rate of the solid and liquid. Although the internal structure of the mushy zone cannot be distinguished, the advancing front can be seen as the moving, half-luster flat interface. However, when the initial $\mathrm{Pb}$ concentration is large, the interface is hard to observe because of the solidification shrinkage and complicated interface shape. Fig. 3(b) shows the microstructure after the solidification shown in Fig. 3(a). The fine grains near the cold wall indicate that solidification was initiated by nucleation at the cold wall (photograph (i)). In the initially undercooled region, the Sn-rich solid solution of the cellular type grew in the direction opposite to the heat flow (photograph (ii)). The crystal spacing is distributed over approximately 10-20 $\mu \mathrm{m}$, and the $\mathrm{Pb}$ becomes concentrated in the gap between the crystals. In the non-undercooled region, cellular and dendritic crystals coexist, and the secondary arm of the dendrite restrains the growth of the adjacent primary arm (photographs (iii) and (iv)). Such a complicated microstructure is formed with the following macroscopic transport phenomena. 
When the alloy melt in the mold was cooled by external cooling, the temperature near the cold wall fell below the liquidus temperature, $218^{\circ} \mathrm{C}$. In Fig. 3(c), the non-uniform undercooling state appears as the shaded zone, and solidification is initiated by heterogeneous nucleation on the cold wall at about $190^{\circ} \mathrm{C}$. The temperature on the cold wall rises about $10^{\circ} \mathrm{C}$ by the recalescence due to the solidification, as shown by $T_{w}$ in Fig. 3(d). The position of the advancing solidification front during this time is also shown in Fig. 3(d). Here, the circles (a-b) show the advancing front in the initially undercooled region, and the triangles (after b') show the front in the non-undercooled region. At a-b, the change rate of the advancing front decreases progressively from a large value at the solidification start. After b', the change rate is almost constant. Consequently, it is considered that $\mathrm{a}-\mathrm{b}$ is free crystal growth driven by undercooling and that the quasi-steady solidification process due to external cooling occurs after b'. On the shift from b to b', the advancing front seems to be stagnant for about one second. However, the stagnation time is extremely short compared with that for aqueous solutions. The reason may be the velocity of free growth is comparatively slow and the recovery of the recalescence temperature field is fast because of the small latent heat of fusion, the high thermal conductivity, and so on.

Figure 4 summarizes the above results for the solidification process of metallic alloy systems. First, by external cooling, a non-uniform undercooling state appears near the cold wall (1). Next, free crystals initiated by nucleation on the cold wall grow in the undercooling region with the temperature rise (2-1). During the free growth, the undercooling state does not easily shift to the equilibrium state because the capture of solute to a solid solution (i.e., a small solute amount is rejected from the solid/liquid interface) may restrain the instability of the crystal surface. The restraint of the interfacial instability would be effective as the distribution coefficient $k_{0}$ becomes larger. Thus, the fattening of the crystal may occur as a relaxation process of the constitutional undercooling remaining in the liquid between the crystals (2-2). After the relaxation ceases, the equilibrium solidification process, in which the liquid between the crystals is completely mixed, can proceed (3). 


\subsection{Free growth of crystals}

Free growth from the undercooling state is important because it is a forerunner to subsequent solidification. Namely, crystals formed in the undercooled region provide a framework for the last morphology of the solid phase, and this process should interrupt quasi-steady heat conduction under external cooling. The characteristic behavior of free growth is discussed below.

The free growth velocity $v$ under a uniform undercooling state can be determined as a function of undercooling magnitude $\Delta T$ and solute concentration $C_{i}$ in the Lipton, Glicksman and Kurz (LGK) model [5,6]. Under a uniform undercooling field, the negative gradient of the temperature ahead of an advancing crystal receives the rejected latent heat and solute, and permits the growth of the crystal. In a non-uniform undercooling field, the temperature gradient is positive; however, it is as sumed that the same growth conditions as in a uniform field can be locally recognized near the crystal tip. The prediction by the LGK model and the experimentally measured results for $\mathrm{Pb}-\mathrm{Sn}$ alloys, represented as a local relationship, are shown in Fig. 5. Here, the free growth velocity $v$ and crystal tip radius $R$ predicted theoretically are represented by the bold curved lines and thin curved lines, respectively. The circles represents the experiment results in $v$. The prediction indicates that $R$ increases from a minimum with the increasing initial solute concentration $C_{i}$. Also, $v$ decreases with the increasing $C_{i}$ under a certain undercooling $\Delta T$. The agreement between the prediction and the experimental results confirms that the LGK model can be approximately applied to non-uniform undercooling fields.

\subsection{Microstructure and microsegregation}

Using Bi-Sn alloy melts of 5.3, 13,31, and $55 \mathrm{wt} \% \mathrm{Bi}$ compositions, solidification experiments were carried out on cases in which undercooling appeared over part or all of a specimen region. Experimental results for each composition are shown as follows in Fig. 6: (a) crystal (primary) spacing and morphology changes, (b) temperature distributions at the solidification start and 
concentration distributions along the crystal center axis, and (c) these distributions on a Bi-Sn phase diagram. Using these results, the microscopic features in the solidified texture for each composition are discussed here.

In the case of $\mathrm{Sn}-31 \mathrm{wt} \% \mathrm{Bi}$, it is clarified from Fig. 6(a) that the crystal morphology changes into granular, cellular and dendritic forms successively in the growth direction. The crystal (primary) spacing increases from about $10 \mu \mathrm{m}$ to $70 \mu \mathrm{m}$ according to the development of side branches. These structural characteristics are shown in detail in Fig. 7: (a) microstructures observed with an optical microscope, (b) back-scattered electron images and (c) line analysis of the $\mathrm{Bi}$ concentration. In Fig. 7(b), the dark part is the Sn-rich phase, and the bright part is the Bi-rich phase. From the line analysis (Fig. 7(c)) corresponding to the image in Fig. 7(b), the cyclic distribution, in which the Bi concentration increases gradually from the crystal center to the side, is recognizable. Temperatures at the solidification start are distributed from $163^{\circ} \mathrm{C}$ at the cold wall to $199^{\circ} \mathrm{C}$ at the 10 - mm point, as shown by the solid circles in Fig. 6(b). The undercooling state appears from the cold wall to the $7.5-\mathrm{mm}$ position at the equilibrium point, since the liquidus temperature for $\mathrm{Sn}-31 \mathrm{wt} \% \mathrm{Bi}$ is about $189^{\circ} \mathrm{C}$. On the phase diagram in Fig. $6(\mathrm{c})$, the undercooling state is represented with the line segment a-b: point a corresponds to the cold wall and point $b$ corresponds to the equilibrium point. Point $c$ corresponds to the point at $10 \mathrm{~mm}$. During the solidification initiated under this temperature condition, the solute concentration captured into the crystal tip (the crystal center axis) decreases along the solidus line in the initially undercooled region (region a-b), and maintains a constant value in the non-undercooled region (region $b-c$ ), as shown by the open circles. The solute concentration captured in the $r$ direction (radial direction of the crystal) is shown in Fig. 8. For the cellular structure $(z=1 \mathrm{~mm})$, the solute concentration decreases slightly from the value (about $15 \mathrm{wt} \%$ ) at the center-axis, then increases up to the eutectic composition. For the dendritic structure $(z=5 \mathrm{~mm})$, the solute concentration increases gradually in the $r$ direction, and the eutectic composition is confirmed at the grain boundary. 
In the case of $\mathrm{Sn}-5.3 \mathrm{w} t \% \mathrm{Bi}$, shown in Fig. $6(\mathrm{~b})$ and (c), the solidification started under the undercooling below the solidus line, so that the initial concentration lies in the solid whole region and massive solidification occurs. In the case of $\mathrm{Sn}-13 \mathrm{wt} \% \mathrm{Bi}$, the cellular structure without secondary arms appears from the position away from the cold wall, and fattens gradually away from the cold wall. This implies that there is a little amount of solute rejected from the crystal surface because the difference between the initial concentration and solidus line is small. In the case of $\mathrm{Sn}-55 \mathrm{wt} \% \mathrm{Bi}$, the solute redistribution is similar to other $\mathrm{Sn}-\mathrm{Bi}$ cases and can be explained by extend ing the metastable liquidus and solidus lines in the phase diagram. In this case, however, the larger amount of rejected solute brings about dendrites with a coarse morphology.

\subsection{Solidification path}

On the basis of experimental results, the solidification paths of the advancing crystal tip and crystal surface can be discussed with the phase diagrams in Fig. 6(c) and Fig. 9. The solidification path of undercooled $\mathrm{Ni}-25 \mathrm{wt} \% \mathrm{Sn}$ droplets has been discussed already by Piccone et al. [7]. In this paper, we consider the time and space change at the interface. The results shown by microscopic observations are summarized as follows.

(1) When the undercooling state is located between the liquidus and solidus lines ( $a-b$ in Fig. 6(c)), and/or between the extension lines, after the small temperature rise $\Delta T_{T}$ due to nucleation on the cold wall, the solid/liquid interface becomes the local equilibrium condition (a' and a"). Continuously, the solid and liquid composition at the crystal tip moves along the solidus and liquidus lines corresponding to the initial undercooling (strictly speaking, both curves take into account the reduction of the equilibrium temperature due to the crystal curvature, as shown by the broken lines, that is, paths a'-b' and a"'-b"). The shape of the crystal tip and its velocity are also determined by the initial undercooling and concentration.

(2) In the region that is not undercooled (b-c), the crystal tip will advance und er the control of the external cooling. So, the advancing front is kept at the equilibrium condition (b' and b") corresponding to the initial composition. 
(3) When the undercooling state is lower than the solidus line, solidification occurs in a massive transformation, such that the concen tration of the solid solution maintains the initial composition. (4) The solidification path of the solute capture in the radial direction of the crystal is shown in Fig. 9: (a) the initially undercooled region; (b) the region not undercooled. In the free growth process, the solute concentration captured at the crystal surface decreas es along the solidus line as the temperature increases due to recalescence. In the fattening process or the equilibrium solidification process, or both, the solute concentration captured at the crystal surface increas es as the temperature drops by external cooling. If the initial composition of the melt is not captured in the radial direction of the crystal, a eutectic structure is formed between the crystals. Otherwise, a simple grain boundary is formed.

\section{CONCLUSIONS}

The solidification process of undercooled alloy melts was studied experimentally, and the following conclusions were obtained.

1. The solidification process is classified into three fundamental processes: (1st stage) free growth with recalescence, (2nd stage) fattening of the crystal with the relaxation of constitutional undercooling, and (3rd stage) equilibrium solidification. However, the free growth and fattening of the crystal does not always occur as separate stages because of the slowness of the free growth velocity compared with the fast recovery of the recalescence.

2. The velocity of free growth depends on the local undercooling and initial concentration, and conforms to the LGK model.

3. Through microscopic observations with SEM/EDS, the solidification path involving the mechanism of solute redistribution during the three fundamental processes is clarified on phase diagrams. 


\section{REFERENCES}

[1] Yoshioka H, Tada Y, Hayashi Y. Acta Mater 2004;52:1515.

[2] Hayashi Y, Kunimine K. Trans Jpn Soc Mech Eng 1992;58-546B:484.

[3] Hayashi Y, Kunimine K, Yoshioka H. Trans Jpn Soc Mech Eng 1995;61-591B:4151.

[4] Hayashi Y, Kunimine K, Takamori J, Yoshida M. Trans Jpn Soc Mech Eng 1996;62-596B:1517.

[5] Lipton J, Glicksman ME, Kurz W. Mater Sci Eng 1984;65:57.

[6] Lipton J, Glicksman ME, Kurz W. Metall Trans 1987;18A:341.

[7] Piccone TJ, Wu Y, Shiohara Y, Flemings MC. Metall Trans 1987;18A:925. 


\section{Figure Captions}

Fig. 1. Solidification process in $\mathrm{H}_{2} \mathrm{O}-\mathrm{NaCl}$ aqueous solutions (diffusion coefficient $k_{0} \approx 0$ ).

Fig. 2. Experimental apparatus for (a) macroscopic observation and (b) microscopic observation.

Fig. 3. Macroscopic observation results obtained for $\mathrm{Sn}-10 \mathrm{wt} \% \mathrm{~Pb}$ : (a) sequence of the advancing solidification front; (b) microstructures observed with an optical microscope; (c) temperature distribution at the solidification start; (d) time changes of the advancing solidification front and temperature at the cold wall.

Fig. 4. Solidification process in alloy melts $\left(0 \ll k_{0}<1\right)$.

Fig. 5. Comparison of experimental data and LGK model on the free growth velocity and crystal tip radius in $\mathrm{Pb}-\mathrm{Sn}$ alloys.

Fig. 6. Microstructure and composition in the $z$-direction (crystal growth direction): (a) crystal arm spacing and morphology changes; (b) temperature distributions at the solidification start and concentration distributions along the crystal center axis; (c) these distributions on a $\mathrm{Bi}$-Sn phase diagram.

Fig. 7. Aspects of the solidified texture in Sn-31wt\%Bi: (a) microstructures observed with an optical microscope; (b) back-scattered electron images filmed with a SEM; (c) line analysis of the Bi concentration using an energy dispersive X-ray spectrometer (EDS) attached to the SEM.

Fig. 8. Concentration distribution in the $r$ direction (crystal radial direction) for $\mathrm{Sn}-31 \mathrm{wt} \% \mathrm{Bi}$.

Fig. 9. Solidification path of the solute capture in the $r$ direction for the (a) initially undercooled region and (b) region not undercooled. 
We are submitting our manuscript entitled "Heat Transfer and Solidification Processes of Alloy Melt with Undercooling-Part I: Experimental results." We would like to submit this paper to the Editor, Professor Richard Wagner. This is one of two revised papers of the manuscript submitted previously (Ms No. A-05-93). We rewrote and revised the paper according to the recommendations of the reviewer, and split the original paper in to two separate papers. This paper is the first (Part I) of the two papers which are being resubmitted individually. In th is paper, we have studied experimentally the complex solidification process, in which the thermodynamically unstable undercooling field collapses by solidification and shifts to a stable system. We hope that this paper is suitable and acceptable for publication.

Please allow us to note here our responses to the reviewer's comments.

First, we would like to thank you very much for the reviewer's helpful comments. According to the reviewer's comments, we split our previous manuscript into two independent papers, and we have revised all paragraphs according to the reviewer's suggestions. The rev isions are as follows. Mandatory Revisions:

(1) In the abstract and in the text the authors mention experiments with $\mathrm{Pb}-\mathrm{Sn}$. However, results are nowhere given in the paper. Therefore, the relationship with the Bi-Sn experiments should be discussed.

(Response)

We have discussed the solidification phenomena as a subject common to many metallic alloy systems. In this study, $\mathrm{Pb}-\mathrm{Sn}$ and $\mathrm{Bi}-\mathrm{Sn}$ with low melting points were used because they are easy to handle. We described the aim of the Pb-Sn experiment and the Bi-Sn experiment in Sec. 2.1 and Sec. 2.2, respectively. Also, we added the experimental results observed using $\mathrm{Pb}-\mathrm{Sn}$ in Sec. 3.1. The free growth velocities shown in Fig. 5 were measured in the $\mathrm{Pb}$ - $\mathrm{Sn}$ experiments.

(2) The introduction contains too many general statements on solidification, which are well known. The text can be shortened and probably combined with Sec. 3 for a more straightforward introduction to the aim of the paper.

(Response)

In accordance with the reviewer's suggestion, we reconsidered the introduction and rewrote it in more straightforward manner by combining it with the description of the solidification process of an aqueous solution in Sec. 3 .

(3) The meaning of Fig. 4 is not ad equately described in the text and in the figure caption. 
(Response)

In accordance with the reviewer's suggestion, we described the meaning of Fig. 5 (old Fig. 4) in the text and in the figure caption.

(4) The reader feels a large gap between Sec. 2 "experimental" and Figures 4, 5. Only indirect results are presented in these figures, which were derived from original data of the solidification process. A figure directly presenting results of the solidification experiments, for example, frames of the advancing solidification front, would be a useful supplement to support better understanding of the subject.

(Response)

In accordance with the reviewer's suggestion, we inserted frames of the advancing solidification front into Fig. 3 and discussed the solidification process to bridge the gap in Sec. 3.1.

(5) A large original of Fig. 6 must be provided for reproduction.

(Response)

In accordance with the reviewer's suggestion, we have attached a large original of Fig. 7 (old Fig. $6)$.

Optional Revisions:

(1) Fig. 3 is not necessary in the context of the paper. Anyway, the mathematical expressions for the various undercooling terms are not given. Fig. 3 and the sentence "Here, the total undercooling" (P. 9) can be omitted. The complete set of model equations is presented in the references.

(Response)

We deleted Fig. 3 and the mathematical expressions, and have presented details of the LGK model in the references.

\section{Hideaki Yoshioka}

Toyama National College of Maritime Technology

Tel.: +81-766-86-5235; Fax: +81-766-86-5110.

E-mail: yoshi@toyama-cmt.ac.jp 

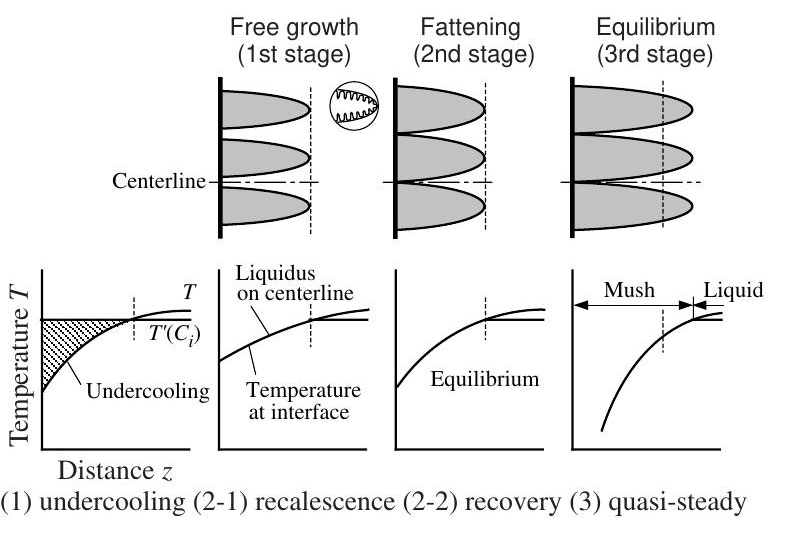

Distance $z$

(1) undercooling (2-1) recalescence (2-2) recovery (3) quasi-steady

Fig. 1

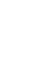


(a)

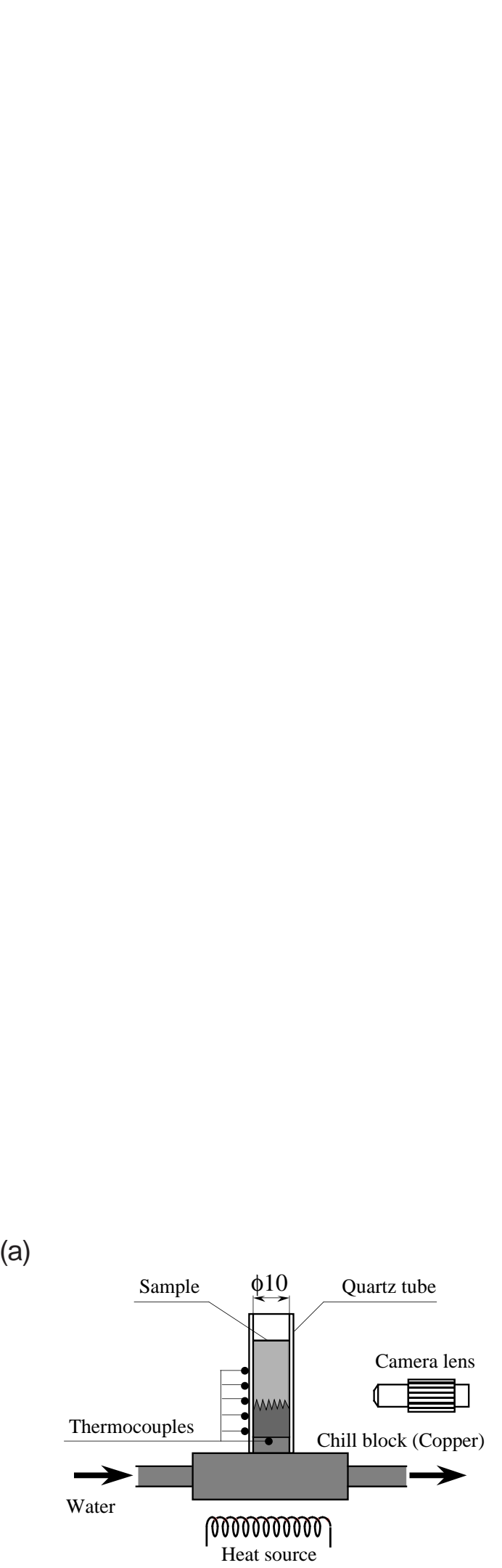

(b)

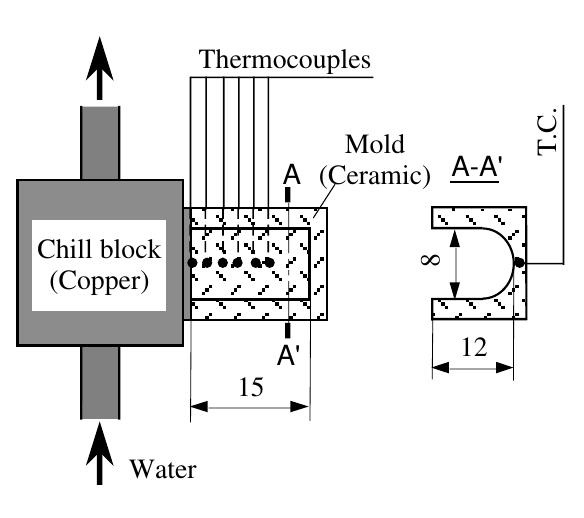

Fig. 2

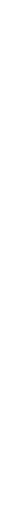




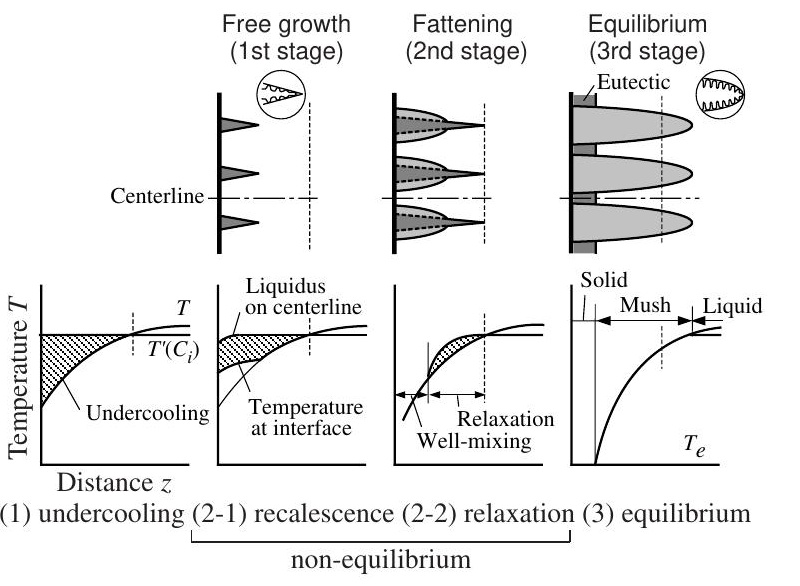

Distance $z$
(1) undercooling (2-1) recalescence (2-2) relaxation (3) equilibrium non-equilibrium

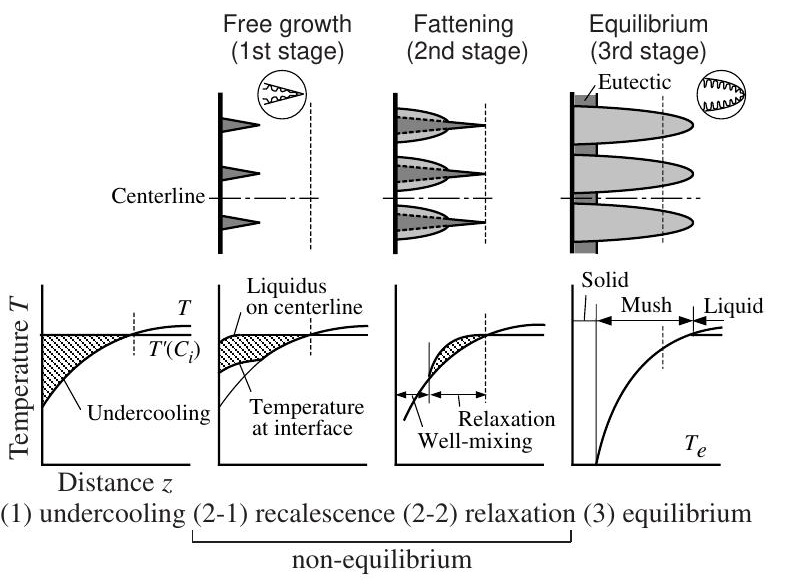

Fig. 4

.


Fig. 6

(a)

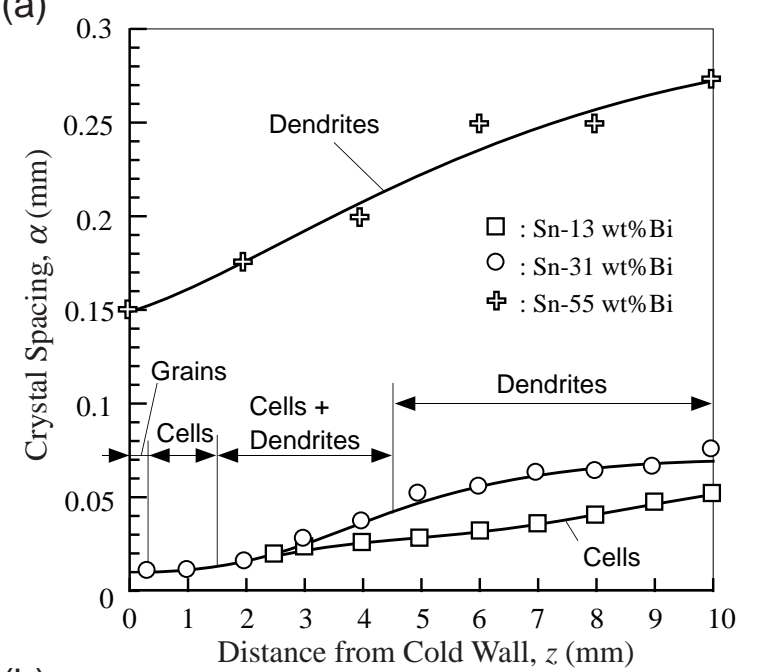

(b)

$$
\text { 过 }
$$

,

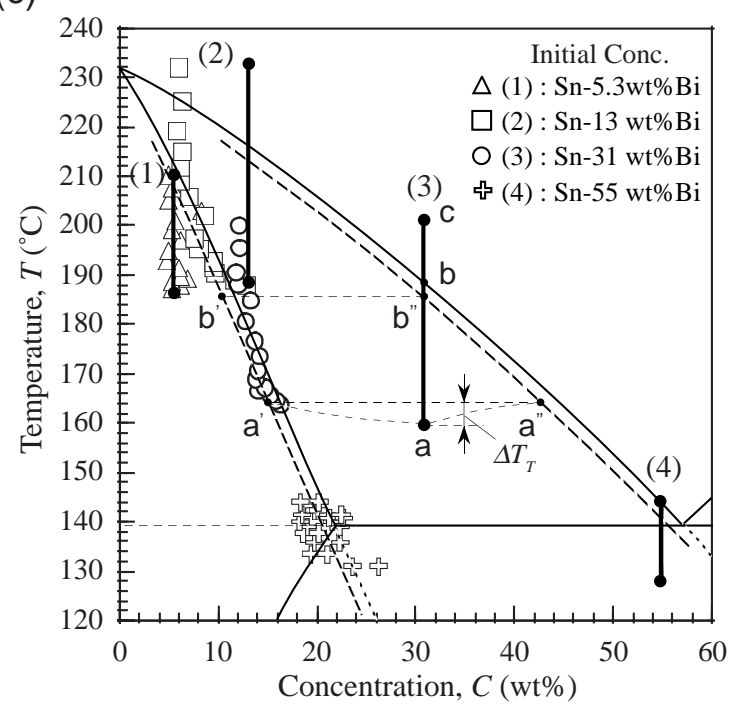


Fig. 7

Growth direction $>$

(a)

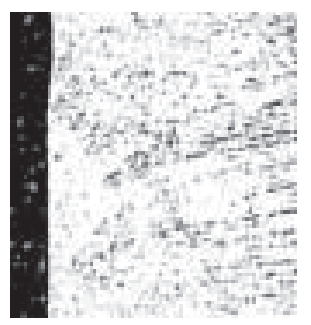

(b)

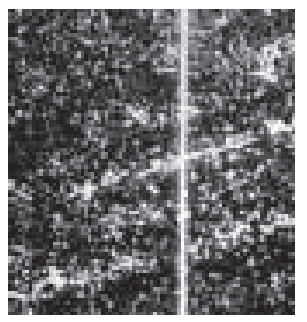

(c)

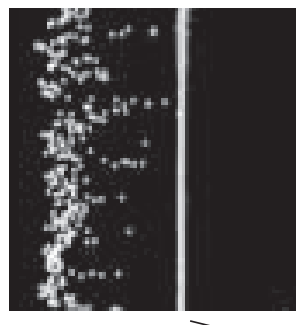

$\mathrm{X}$-ray indensity (Bi)

$z=0.3 \mathrm{~mm}$
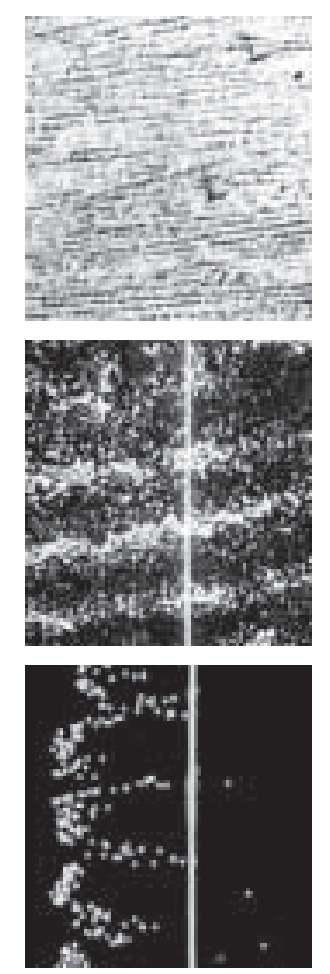
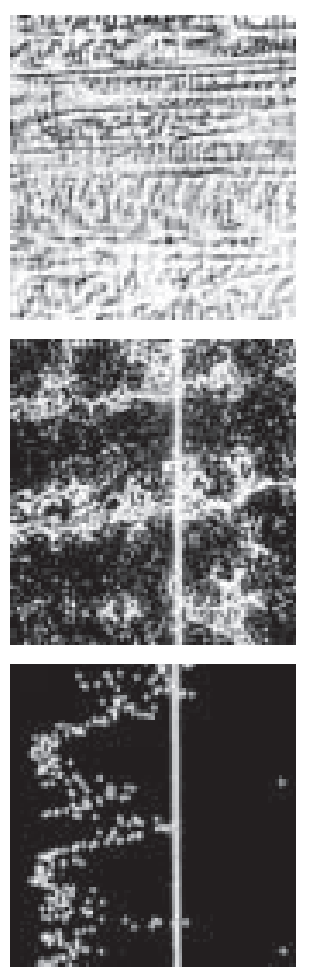
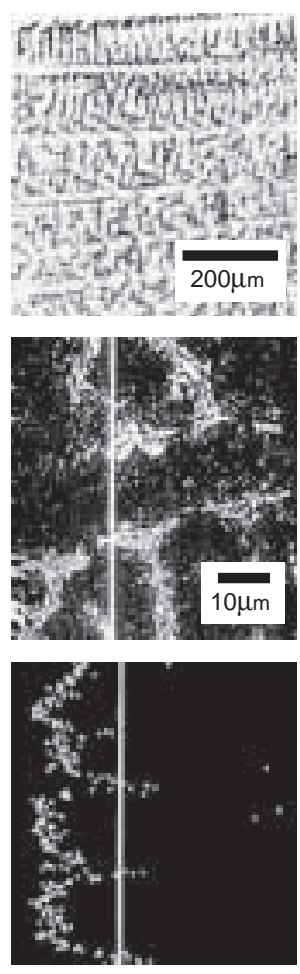
(a)

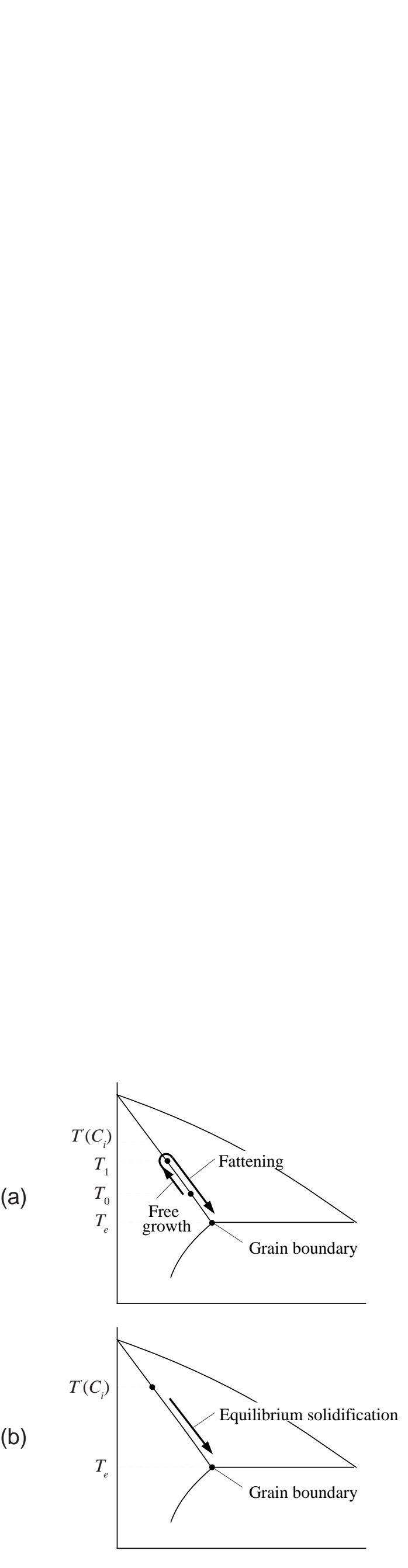

(b)

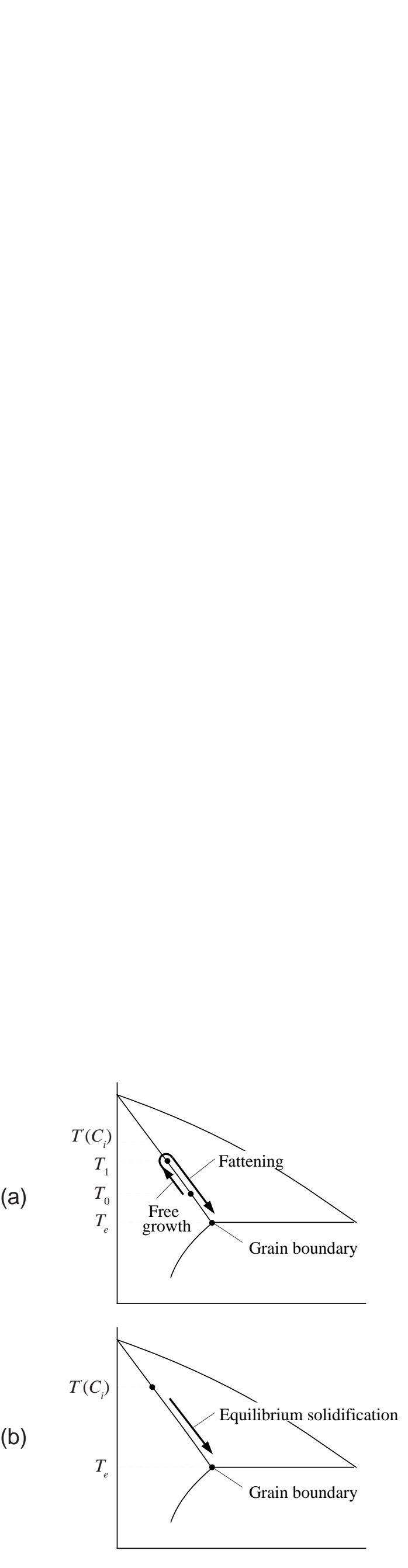

Fig. 9

9

fication
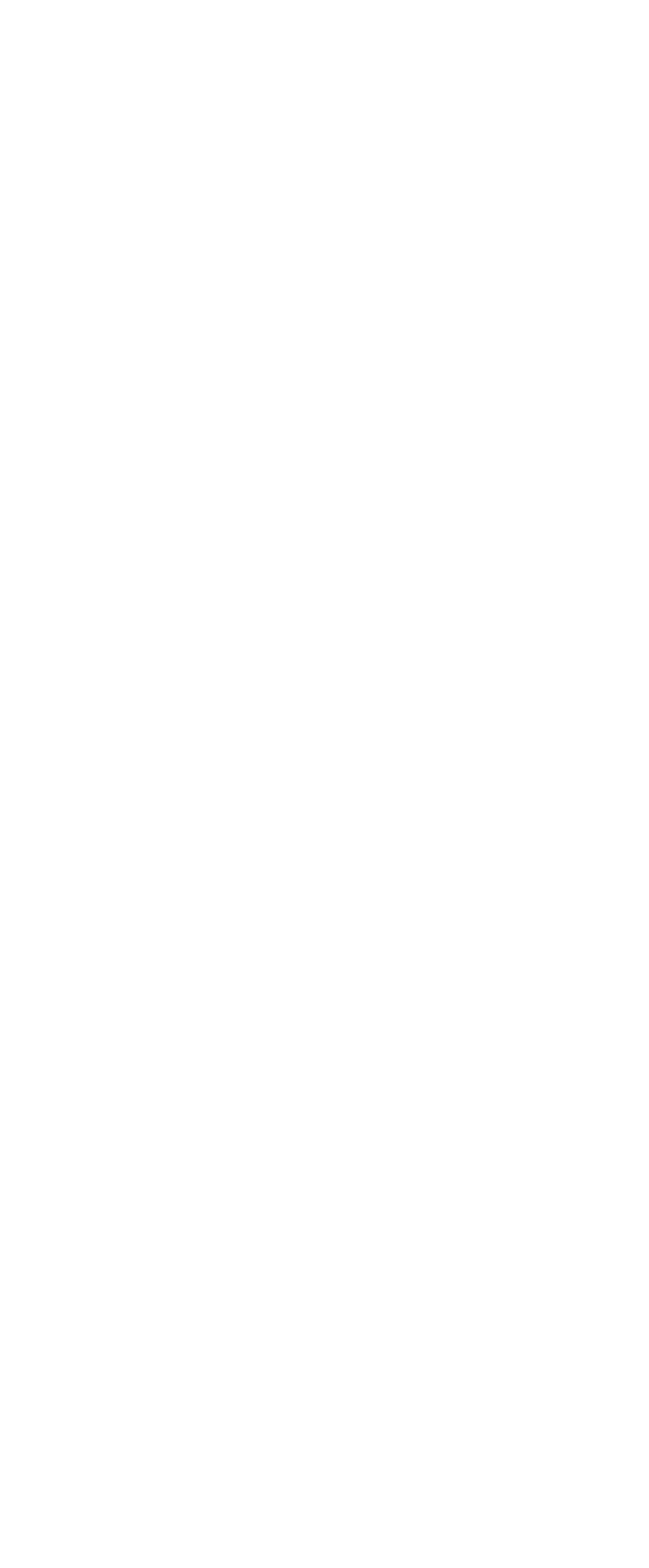